\title{
Early pathological changes in the parkinsonian brain demonstrated by diffusion tensor MRI
}

\author{
K Yoshikawa, Y Nakata, K Yamada, M Nakagawa
}

See Editorial Commentary, p 352

J Neurol Neurosurg Psychiatry 2004;75:481-484. doi: 10.1136/jnnp.2003.021873

\begin{abstract}
Objective: To determine whether the fractional anisotropy (FA) of magnetic resonance diffusion tensor imaging is decreased in the nigrostriatal projection in parkinsonian patients.

Methods: FA values were compared in the extrapyramidal system of 12 patients with Parkinson's disease and eight age matched normal controls.

Results: Patients with Parkinson's disease had significantly decreased FA in the region of interest along a line between the substantia nigra and the lower part of the putamen/ caudate complex, in which most of the nigrostriatal dopaminergic neurones are included. Loss of FA in this region was obvious even during the early clinical stages of Parkinson's disease.

Conclusions: Assuming that the loss of FA parallels the neuronal change in the brain, the results are consistent with the view that more than half the dopaminergic neurones in the nigrostriatal projection are lost before the onset of Parkinson's disease. Close comparison of FA in the basal ganglia may contribute to the early diagnosis of Parkinson's disease.
\end{abstract}

$\mathrm{T}$ he discovery of the selective loss of dopaminergic neurones projecting from the substantia nigra in the midbrain to the neostriatum in patients with idiopathic Parkinson's disease has led to the use of l-dopa treatment, which markedly improves the prognosis of parkinsonian patients. ${ }^{12}$

According to pathological studies, at least $70-80 \%$ of dopaminergic neurones were lost before the onset of Parkinson's disease. ${ }^{3}$ A significant amount of dopaminergic neurone loss during the early stages of Parkinson's disease was also suggested by functional imaging of these neurones using positron emission tomography (PET) with L- $\left[{ }^{18} \mathrm{~F}\right]-$ fluorodopa ${ }^{4}$ or single photon emission computer tomography (SPECT) with $\left[{ }^{123} \mathrm{I}\right]-\beta C I T{ }^{5}$

Recently, magnetic resonance (MR) diffusion tensor imaging of the brain has allowed visualisation of neuronal projections in the central nervous system, ${ }^{6}$ or estimation of the neuronal changes in the white matter of either normal subjects $^{7}$ or patients with neurological diseases such as amyotrophic lateral sclerosis ${ }^{8}$ and multiple sclerosis, ${ }^{9}$ through a decrease in the fractional anisotropy (FA) value derived from the MR tensor image. In the present study, we compared FA values in the extrapyramidal system of normal subjects with those of parkinsonian patients.

\section{METHODS}

Twelve patients with Parkinson's disease (mean (SD) age, 71.3 (7.7) years) were compared with eight age matched normal subjects (70.1 (8.4) years). Seven patients with progressive supranuclear palsy (PSP) (74.6 (7.3) years) were also studied. As patients with PSP show brain atrophy, including atrophy of the basal ganglia, it is suggested that they may serve as positive controls for FA change showing a robust decrease.

Parkinson's disease was diagnosed using the UK Brain Bank criteria. ${ }^{10}$ The patients were separated into two groups, depending on the Hoen and Yahr rating scale ${ }^{11}$ : patients in stage I or II were classified as the earlier group $(\mathrm{n}=7 ; 70.0$ (3.7) years), and those in stage III or more were classified as the advanced group $(\mathrm{n}=5 ; 73.2$ (2.4) years).

The diagnosis of PSP was according to the NINDS-SPSP criteria. ${ }^{12}$ Normal subjects were examined by neurologists, and no abnormality was confirmed.

Informed consent for the study was obtained from each subject before the examination. MR imaging (MRI) was done with a $1.5 \mathrm{~T}$ imager ("Gyroscan Intera", Philips Medical Systems, Best, Netherlands), between August 12001 and October 31 2002. In addition to conventional Tl weighted images (repetition time (TR) $611 \mathrm{~ms}$, echo time (TE) $13 \mathrm{~ms}$ ) and T2 weighted images (TR 4754, TE 100), diffusion tensor images (DTI) were obtained. The imaging sequence of DTI was as follows: field of view $230 \times 230 \mathrm{~mm}$; matrix $128 \times 37$ with SENSE; SENSE reduction factor $=2($ matrix is $128 \times 74$ equivalent); TR/TE $=6000 / 88$; flip angle $=90^{\circ}$; two $\mathrm{b}$ values $\left(0\right.$ and $\left.800 \mathrm{~s} / \mathrm{mm}^{2}\right)$; 36 slices; $3 / 0 \mathrm{~mm}$ slice/gap. Diffusion sensitisation was done in six directions. ${ }^{13}$

FA values of 15 regions of interest (ROI) from a single hemisphere were compared between normal subjects and the patient groups. The investigators (KY and YN) were blinded to the patient's name and group. ROIs were determined (see below) on conventional MRI, and the mean FA values in the corresponding DTI were measured.

According to the landmarks, four ROIs were allocated on MRI plane " $\mathrm{b}$ " and " $\mathrm{c}$ " (fig lA, lC, and lD): oval ROIs sized $41 \mathrm{~mm}^{2}$ in the caudate head, putamen, nucleus ventralis lateralis, and subcortical white matter of the premotor cortex (area 6).

The remaining 11 ROIs were set on an axial MR image in the basal ganglia (plane " $\mathrm{a}$ " in fig $\mathrm{IA}$ and $\mathrm{lB}$ ). The plane immediately below the AC-PC line roughly included structures such as the substantia nigra, subthalamic nucleus, and the lower part of the globus pallidus, putamen, and caudate nucleus. ${ }^{14}$ This plane is also suggested to include most of the nigrostriatal neurones. ${ }^{15}{ }^{16}$ Eleven oval ROIs sized $20 \mathrm{~mm}^{2}$ were arrayed along a line between the substantia nigra and the caudate/putamen complex at the same interval so that the ROIs covered the majority of the dopaminergic neurones between the structures (fig 1B).

To avoid the influence of motion, the subject's head was firmly fixed to the holder during acquisition. In addition, we

Abbreviations: DTI, diffusion tensor image; FA, fractional anisotropy 


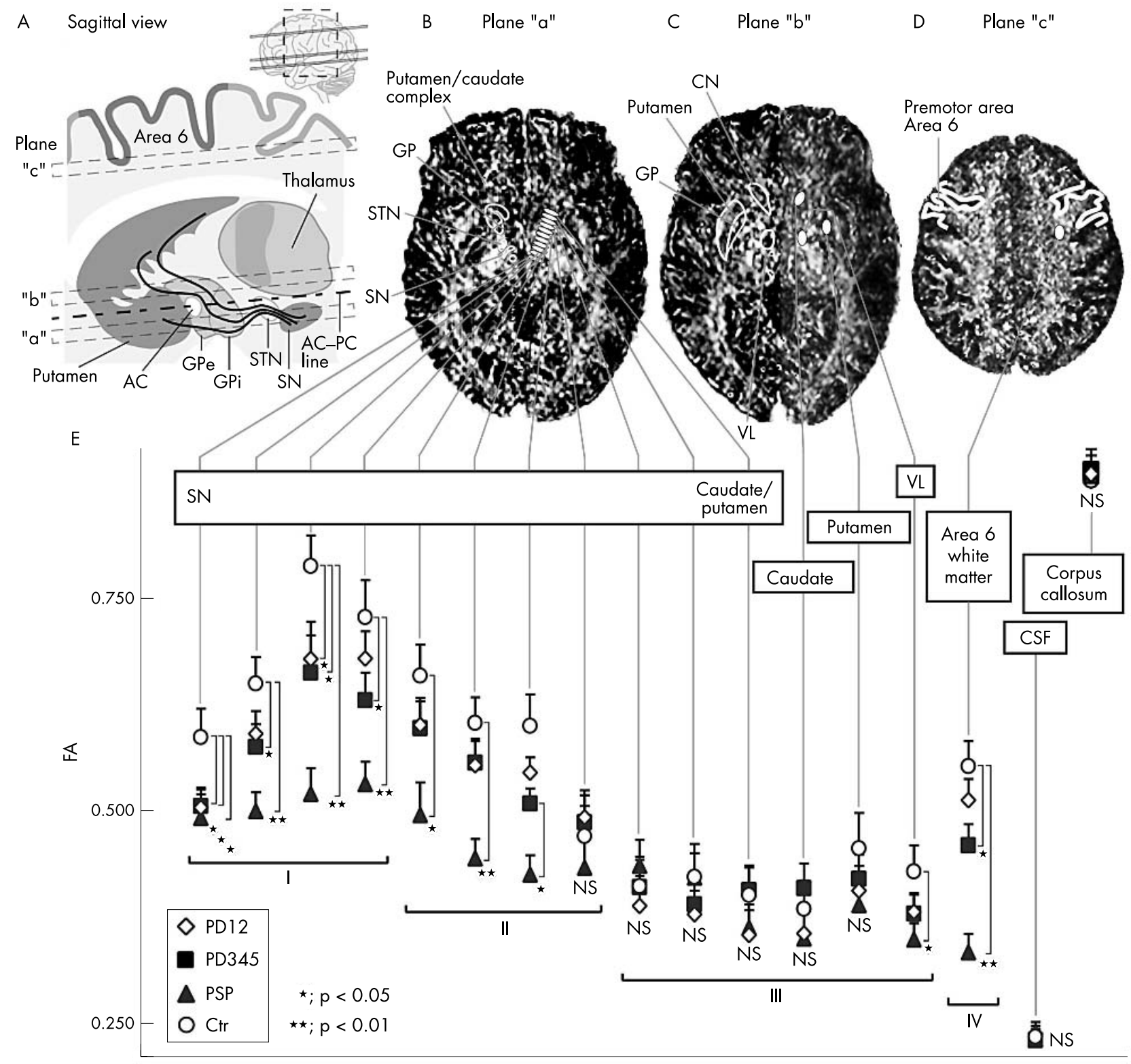

Figure 1 (A) Anatomy of the extrapyramidal system in the paramedian sagittal view. Localisation of axial planes of magnetic resonance imaging (MRI) is indicated by dotted squares. Fibres of nigroneostriatal projection, which are selectively lost in Parkinson's disease, are illustrated by black lines originating from the substantia nigra. (B-D) Fractional anisotropy (FA) images derived from diffusion tensor images are shown with 11 small ROls and three large ROIs. (E) FA in the extrapyramidal system of normal subjects and patients. Mean FA values of the ROls in each group are indicated by figures with error bars. FA values in patients with progressive supranuclear palsy (PSP) were decreased in most of the ROls except for those in the neostriatum. Parkinsonian patients showed a significant decrease in the FA values of the subthalamic ROI beside the substantia nigra. The FA values in the white matter of the premotor cortices were significantly smaller than controls in PSP and patients with advanced Parkinson's disease. I, II, III, and IV in fig $1 \mathrm{E}$ indicate the four regions in the extrapyramidal system. AC, anterior commissure; CN, caudate nucleus; Ctr, age matched normal subjects as control; GP, globus pallidus; GPe, globus pallidus lateral segment; GPi, globus pallidus medial segment; PC, posterior commissure; PD12, Parkinson's disease in the early stage group; PD345, Parkinson's disease in the advanced stage group; PSP, progressive supranuclear palsy; ROI, region of interest; SN, substantia nigra; SNc, substantia nigra pars compacta; SNr, substantia nigra pars reticulate; STN, subthalamic nucleus; VL, nucleus ventralis lateralis.

monitored the FA of the cerebrospinal fluid (CSF) in the lateral ventricles and the splenium of the corpus callosum. If the FAs in these structures were lower than the mean value minus 2 SD of the normal subjects, we rejected the patient.

\section{Analyses}

Statistical evaluations were done using SPSS 11.0J for Windows. Multiple comparisons of the 38 hemispheres in three patient groups with 16 hemispheres of normal subjects as a control were undertaken using Dunnett's method. Statistical significance was defined by a probability $(p)$ value of 0.05 .

\section{RESULTS}

The mean FA values of all the ROIs are illustrated in fig IE. The FAs of either the CSF or the corpus callosum were very consistent among the subjects, and no patient was rejected.

In patients with PSP, a significant decrease in the FA values was observed in most of the subthalamic structures 
Table 1 Fractional anisotropy (FA) values in five circuits of the extrapyramidal system

\begin{tabular}{|c|c|c|c|c|c|}
\hline & \multicolumn{5}{|c|}{ Circuits (corresponding regions) } \\
\hline & P (II+III+IV) & First (II+III) & Second (II) & Third (I+II+III) & Fourth $(\mathrm{I}+\mathrm{II}+\mathrm{III}+\mathrm{IV})$ \\
\hline Control & 0.490 & 0.483 & 0.583 & 0.542 & 0.542 \\
\hline PD12 & 0.451 & 0.445 & 0.548 & $0.493^{*}$ & $0.434^{*}$ \\
\hline PD345 & 0.456 & 0.456 & 0.537 & $0.495^{*}$ & $0.493^{*}$ \\
\hline PSP & $0.403^{* *}$ & $0.410^{* *}$ & $0.449^{* *}$ & $0.439 * *$ & $0.432^{* *}$ \\
\hline
\end{tabular}

Parkinsonian patients showed a significant decrease in FA values in the third and fourth accessory circuits, regardless of clinical stage. ${ }^{*} p<0.05,{ }^{* *} p<0.01$.

P, principal circuit; PD12, Parkinson's disease in the early stage group; PD345, Parkinson's disease in the advanced stage group; PSP, progressive supranuclear palsy.

$(-16.2 \%$ to $34.1 \%, \mathrm{p}<0.01$ to 0.05$)$. Subcortical white matter also showed a severe decrease $(-39.6 \%, \mathrm{p}<0.01)$.

In patients with Parkinson's disease, FA values were significantly low in ROIs beside the substantia nigra $(-10.5 \%$ to $16.0 \%, \mathrm{p}<0.05)$. In advanced cases, subcortical white matter also showed low FA values compared with the normal subjects $(-16.9 \%, \mathrm{p}<0.05)$. FA values in the putamen, caudate nucleus, or nucleus ventralis lateralis showed no significant decrease in either subgroup.

For further evaluation, we compared the regional change of FA in the extrapyramidal system. The system consisted of one principal striatal circuit and four accessory circuits. ${ }^{17}$ Accordingly, we divided the 15 ROIs into four regions (regions I to IV in fig $\mathrm{IE}$ ), and assigned the regions to each neural circuit: the principal circuit $(\mathrm{P})$-which included the cortex, neostriatum, globus pallidus, and thalamus—could be estimated by ROIs in regions II, III, and IV. In the same way, the first accessory circuit corresponded to regions II and III, the second to region II, the third to regions I, II, and III, and the fourth to all regions. We then compared the FA values of each circuit in normal subjects with those in the patient groups.

In patients with PSP, the FA values were low in all of the circuits compared with the normal subjects. The patients with Parkinson's disease showed a significant decrease in the third and fourth circuits where the nigrostriatal projections were involved, regardless of the clinical stage of the disease (table 1).

\section{DISCUSSION}

Duguid et al reported that patients with Parkinson's disease showed histological changes in the midbrain which can be detected as subtle alterations around the substantia nigra on conventional MRI. ${ }^{18}$ These differences were too small to help in distinguishing parkinsonian brains from normal brains with high reproducibility in routine clinical imaging.

After the study, advanced MR techniques such as diffusion weighted imaging and magnetisation transfer ratios were made available for clinical use and immediately applied to the diagnosis of Parkinson's disease. However, these attempts failed to distinguish patients with Parkinson's disease from normal subjects. ${ }^{19} 20$

The FA value is derived from eigenvalues of the diffusion tensor and is a measure of the anisotropy of water diffusion in tissue: completely isotropic diffusion has an FA of 0 , whereas in highly anisotropic diffusion the FA approaches 1. As far as white matter is concerned, uniformity of fibre bundles is the primary issue: pyramidal tract or corpus callosum, in which unidirectional fibres are dominant, will show high FA values. ${ }^{21}$ A decrease in FA of these structures may be a sensitive indicator of histological abnormality, even if the values were derived from normal-appearing tissue by conventional MRI.22 At present, the pathological interpretation of FA reduction in the brain with neurodegenerative disease is not clear, and may be caused by-among other things-neuronal loss, gliosis, or demyelination..$^{7-9}$

According to this assumption, PSP patients were expected to show a robust decrease of FA in these structures, and the change was confirmed in this study. Similarly, patients with Parkinson's disease may show low FA values in the nigrostriatal projection, which is selectively lost in the disease. As the projections consist of so few and such small dopaminergic neurones that they cannot be allocated by conventional MRI or other techniques such as fibre tracking, ${ }^{623}$ we spread arrays of ROIs in subthalamic structures, where the major part of the nigrostriatal projections would be involved. ${ }^{15}$

The present results showed that the parkinsonian patients had decreased FA values in two striatal circuits in which dopaminergic neurones are included, and the change was evident regardless of clinical stage. Assuming that the loss of FA parallels the neuronal change in the brain, our results corresponded well to the view that $70-80 \%$ of dopaminergic neurones are lost before the onset of Parkinson's disease.

Our study also revealed a significant change in subcortical white matter among the cases of advanced Parkinson's disease. This might be a result of extended damage in the extrapyramidal system including the corticostriatal and thalamocortical projections.

Finally, the results suggested that FA values potentially contribute to the diagnosis of neurodegenerative disorders with normal appearing brain by conventional MRI, although the correlation between the FA and pathological changes in brain tissue is a primary concern for further studies.

\section{ACKNOWLEDGEMENTS}

This work was supported by a grant for young researchers from the Ministry of Education, Culture, Sports, Science and Technology of Japan (14770294).

\section{Authors' affiliations}

K Yoshikawa, Y Nakata, M Nakagawa, Department of Neurology, Research Institute for Neurological Diseases and Geriatrics, Kyoto Prefectural University of Medicine, Kyoto, Japan

K Yamada, Department of Radiology, Kyoto Prefectural University of Medicine

Competing interests: none declared

Correspondence to: Dr Kenji Yoshikawa, 465 Kawaramachi-Hirokoji, Kamigyo-ku, Kyoto 602-8566, Japan; kyoshika@koto.kpu-m.ac.jp

Received 25 June 2003

In revised form 11 September 2003

Accepted 1 November 2003

\section{REFERENCES}

1 O'Reilly S, Loncin M, Cooksey B. Dopamine and basal ganglia disorders. Neurology 1965;15:980-4. 
2 Cotzias GC, Papavasiliou PS, Gellene R. Modification of Parkinsonismchronic treatment with L-dopa. N Engl J Med 1969;280:337-45.

3 Riederer $\mathbf{P}$, Wuketich S. Time course of nigrostriatal degeneration in Parkinson's disease. A detailed study of influential factors in human brain amine analysis. J Neural Transm 1976;38:277-301.

4 Leenders KL, Palmer AJ, Quinn N, et al. Brain dopamine metabolism in patients with Parkinson's disease measured with positron emission tomography. J Neurol Neurosurg Psychiatry 1986;49:853-60.

5 Innis RB, Seibyl JP, Scanley BE, et al. Single photon emission computed tomographic imaging demonstrates loss of striatal dopamine transporters in Parkinson disease. Proc Natl Acad Sci USA 1993;90:11965-9.

6 Conturo TE, Lori NF, Cull TS, et al. Tracking neuronal fiber pathways in the living human brain. Proc Nat Acad Sci USA 1999;96:10422-7.

7 Abe O, Aoki S, Hayashi N, et al. Normal aging in the central nervous system: quantitative MR diffusion-tensor analysis. Neurobiol Aging 2002;23:433-41.

8 Ellis CM, Simmons A, Jones DK, et al. Diffusion tensor MRI assesses corticospinal tract damage in ALS. Neurology 1999;53:1051-8.

9 Tievsky AL, Ptak T, Farkas J. Investigation of apparent diffusion coefficient and diffusion tensor anisotropy in acute and chronic multiple sclerosis lesions. Am J Neuroradiol 1999;20:1491-9.

10 Hughes AJ, Daniel SE, Kliford L, et al. Accuracy of clinical diagnosis of idiopathic Parkinson's disease: a clinico-pathological study of 100 cases. J Neurol Neurosurg Psychiatry 1992:55:181-4.

11 Hoehn MM, Yahr MD. Parkinsonism: onset, progression, and mortality. Neurology 1967; 17:427-2.

12 Litvan I, Agid Y, Calne D, et al. Clinical research criteria for the diagnosis of progressive supranuclear palsy (Steele-Richardson-Olszewski syndrome): report of the NINDS-SPSP international workshop. Neurology 1996;47:1-9.
13 Yamada K, Kizu O, Mori S, et al. Brain fiber tracking with clinically feasible diffusion-tensor MR imaging: initial experience. Radiology 2003;227:295-301.

14 Talairach J, Tournoux $P$, eds. Three-dimensional atlas of a human brain. In: Co-planar stereotaxic atlas of the human brain. Three-dimensional proportional system: an approach to cerebral imaging, 1st ed. Stuttgart: Georg Thieme Verlag, 1988.

15 Anden NE, Carlsson A, Dahlstrom A, et al. Demonstration and mapping out of nigro-neostriatal dopamine neurons. Life Sci 1964:3:523-30.

16 Carpenter MB, Peter P. Nigrostriatal and nigrothalamic fibers in the rhesus monkey. J Comp Neurol 1972;144:93-115.

17 Alexander GE. Basal ganglia-thalamocortical circuits: their role in control of movements. J Clin Neurophysiol 1994;11:420-31.

18 Duguid JR, De La Paz R, DeGroot J. Magnetic resonance imaging of the midbrain in Parkinson's disease. Ann Neurol 1986;20:744-7.

19 Schocke MF, Seppi K, Esterhammer R, et al. Diffusion-weighted MRI differentiates the Parkinson variant of multiple system atrophy from PD. Neurology 2002;58:575-80.

20 Hanyu $\mathrm{H}$, Asano T, Sakurai $\mathrm{H}$, et al. Magnetisation transfer measurements of the subcortical grey and white matter in Parkinson's disease with and without dementia and in progressive supranuclear palsy. Neuroradiology 2001;43:542-6.

21 Le Bihan D, Turner R, Douek $P$, et al. Diffusion MR imaging: clinical applications. Am J Roentgenol 1992;159:591-9.

22 Lu S, Ahn D, Johnson G, et al. Peritumoral diffusion tensor imaging of highgrade gliomas and metastatic brain tumors. Am J Neuroradiol 2003; 24:937-41

23 Mori S, Crain BJ, Chacko VP, et al. Three-dimensional tracking of axonal projections in the brain by magnetic resonance imaging. Ann Neurol 1999;45:265-9. 